\title{
1. The nature of organizational neuroscience
}

Organizations can be construed as social cognitive systems populated by people who gather, compute, analyze, and interpret information, and interact with one another. These social cognitive systems cannot be studied without reference to the neural substrates that underlie cognitions. Thus, this chapter presents the nascent field of organizational neuroscience to the reader. In doing so, it provides a working definition of organizational neuroscience, discusses its interdisciplinary nature, explores its levels of analysis, and identifies some of the research questions it could help elucidate.

\section{UNDERSTANDING ORGANIZATIONAL NEUROSCIENCE}

In recent years, there has been increasing interest in applying neuroscientific methods and techniques to the study of organizational phenomena (Butler and Senior, 2007; Lee and Chamberlain, 2007; Senior, Lee, and Butler, 2011; Becker and Cropanzano, 2010; Beugré, 2010; Becker, Cropanzano, and Sanfey, 2011; Butler, 2014). Beugré (2010) introduced the construct of neuro-organizational behavior, which he defined as the study of the impact of the brain on behavior that occurs in organizations (p. 289). Likewise, Butler and Senior (2007), Lee and Chamberlain (2007), and Senior et al. (2011) introduced the field of organizational cognitive neuroscience to explain the role of neuroscience in human behavior in organizations. Lee and Chamberlain (2007, p. 22) defined organizational cognitive neuroscience as "the study of the processes within the brain that underlie or influence human decisions, behaviors, and interactions either a) within organizations or b) in response to organizational manifestations or institutions". This definition is similar to the one provided by Butler and Senior (2007) who conceived of organizational cognitive neuroscience as the use of "neuroscientific methods to analyze and understand human behavior within the applied 
setting of organizations. This application may be at the individual, group, organizational, and interorganizational levels" (p. 8).

Lee, Senior, and Butler (2012b) make a distinction between organizational neuroscience $(\mathrm{ON})$, social cognitive neuroscience $(\mathrm{SCN})$, and organizational cognitive neuroscience (OCN). Specifically, they contend that organizational neuroscience focuses on brain anatomy and structures, whereas SCN and OCN deal with multiple levels of analysis and are interested in the interplay between biological systems and cognitions. The authors acknowledge an overlap between organizational neuroscience and SCN and OCN. Particularly, they argue that "OCN is symbiotic with $\mathrm{ON}$ as well as $\mathrm{SCN}$, to form a detailed theoretical framework that helps scholars to understand the complexities of the social behavior that occurs within organizations" (p. 923).

In this book, I consider that the distinction between OCN and organizational neuroscience is a superficial one because organizational neuroscience does not limit itself to the description of neural anatomy. Rather, it encompasses both a description of brain structures as well as an understanding of the neural mechanisms that substantiate cognitions. My argument is that both organizational neuroscience and organizational cognitive neuroscience study the same phenomena and use the same research tools. For example, the neural basis of topics such as decision making, emotions, cognitions, trust, cooperation, leadership, and ethics are studied by both disciplines using the same neuroscientific methods.

Becker and Cropanzano (2010) coined the term organizational neuroscience, which they conceive "as a deliberate and judicious approach to spanning the divide between neuroscience and organizational science" (p. 1055). They suggest that "existing theories of organizational behavior can be enhanced by incorporating the findings and themes from neuroscience regarding how the brain produces cognition, attitudes, and behaviors" (ibid.). Becker et al. (2011, p. 937) view organizational neuroscience as an "interpretive framework that sheds new light on existing problems as well as highlighting problems that otherwise might not have been considered." Thus, organizational neuroscience could help to answer questions that current organization science research cannot.

The three terms, organizational cognitive neuroscience, neuroorganizational behavior, and organizational neuroscience, have been used to describe the same field. Although this may seem awkward, it is hardly surprising because organizational neuroscience does not even exist as a unified field. Thus, the aim of this book is to contribute to the development of a new field that blends together neuroscience and organizational science. To this end, it is important to consider a unified appellation of the field - common sense and human practice dictate that 
a new baby must be named. Organizational scholars cannot create a field combining neuroscience and the organization sciences if they do not know what this new field is called or cannot delineate it. As a consequence, I make a modest effort to provide a first step in this direction.

In doing so, I favor the term organizational neuroscience used by Becker and Cropanzano (2010) and Becker et al. (2011) to encompass what I described as neuro-organizational behavior (Beugré, 2010) and what Butler and Senior (2007) called organizational cognitive neuroscience or social cognitive neuroscience. I favor this term for two main reasons. First, the term organizational neuroscience tends to be broader than organizational cognitive neuroscience, where the latter could be misleading. Indeed, organizational cognitive neuroscience may appear at first glance to favor conscious efforts made by individuals and overlook social aspects, emotions, or unconscious phenomena. The term organizational neuroscience is also broader than the term neuro-organizational behavior because neuro-organizational behavior could be limited to human behavior in existing organizations, and overlook the application of neuroscience to fields such as entrepreneurship, management information systems, and strategic management.

Second, the term organizational neuroscience falls under the same categories as the terms neuroeconomics and neuromarketing. These latter fields combine the tools of neuroscience with existing theories and models of economics or marketing. Thus, the field of organizational neuroscience aims to combine tools and techniques of neuroscience with organizational science theories.

To make organizational neuroscience a coherent field, organizational scholars must provide a clear definition of the field and identify pertinent research questions that can only be explored by this nascent field. Indeed, "a scientific concept has meaning only because scientists mean something by it. The meaning is scientifically valid only if what they intend by it becomes actual: problems are solved and intentions are fulfilled as inquiry continues" (Kaplan, 1964, p. 46). I therefore define organizational neuroscience as the field that integrates the use of neuroscientific methods and techniques to the study of organizational phenomena. Defined as such, organizational neuroscience is a multidisciplinary field.

\section{ORGANIZATIONAL NEUROSCIENCE AS A MULTIDISCIPLINARY FIELD}

Organizational neuroscience can be construed as a multidisciplinary field that draws from disciplines such as neuroscience, neuroeconomics, social 
cognitive neuroscience, cognitive psychology, and neuroscience. The multidisciplinary nature of organizational neuroscience was advocated by Beugré (2010) who introduced a neuro-organizational behavior paradigm, which he described as a multidisciplinary discipline that draws its knowledge and methods from cognitive psychology, neuroeconomics, neuroscience, organizational behavior, and social cognitive neuroscience. In the following paragraphs, I explore the extent to which each of these five disciplines contributes to the nascent field of organizational neuroscience. Other disciplines not identified here could also benefit organizational neuroscience but I focus on the five illustrated in Figure 1.1.

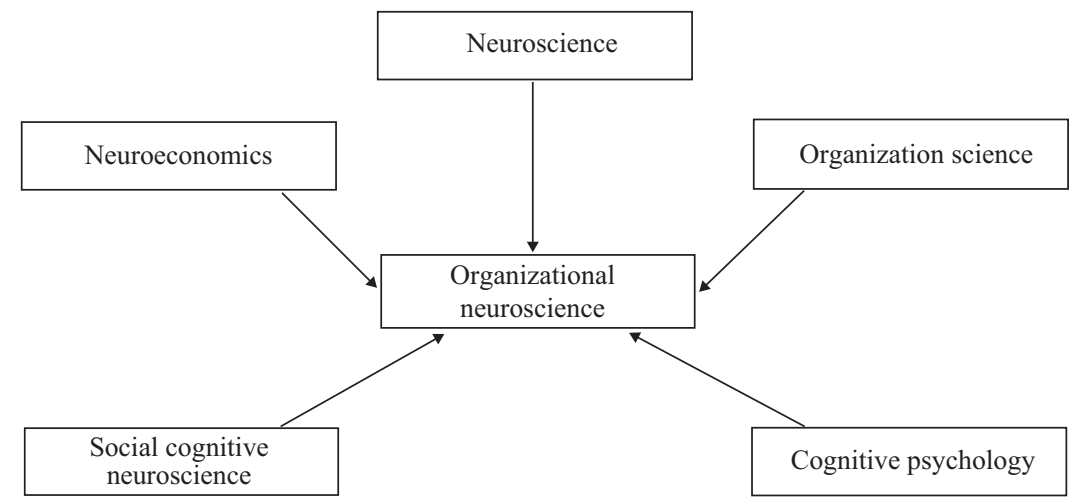

Figure 1.1 Disciplines contributing to organizational neuroscience

\subsection{Neuroscience}

Neuroscience is the study of the brain, its structure, functions, and how it affects human behavior. Neuroscience also helps our understanding of how to repair brain structures and restore brain functions when they are impaired. Neuroscience itself is an interdisciplinary approach stemming from biology, chemistry, mathematics, medicine, physics, and psychology. As Mukamel and Fried (2012, p. 511) put it, "the ultimate goal of neuroscience research is to understand the operating mechanism of the human brain and to exploit this understanding to devise methods for repair when it malfunctions."

Kable (2011) identified three ways in which neuroscience can inform our understanding of human cognition and behavior: (1) the use of association tests; (2) the use of tests of necessity; and (3) the use of tests of sufficiency. First, the use of tests of association involves observing or 
experimentally manipulating psychological states or behavior, simultaneously measuring neural activity, and examining correlations between the two. Second, using a test of necessity involves disrupting neural activity and showing that this manipulation impairs a specific behavior or psychological function. Third, the use of tests of sufficiency involves enhancing neural activity and showing that this manipulation results in a specific behavioral or psychological state. Kable's conceptualization could encompass the use of neuroscientific tools such as functional magnetic resonance imaging (fMRI), electroencephalography (EEG), magnetoencephalography (MEG) and transcranial magnetic stimulation (TMS) to investigate particular organizational phenomena. Neuroscience can help develop "basic processing models that can be used to generate predictions about individual and group performance" (Paulus et al., 2009, p. 1085).

"Neuroscience holds great promise for advancing organizational theories and practice" (Cropanzano and Becker, 2013, p. 310), particularly as "neuroscientific methods provide the most reliable window into the unconscious brain that is currently available" (ibid.). Although neuroscience can provide useful knowledge for organizational scholars, the potential for the misuse of its methods is real. We are already witnessing the overselling of the promise of neuroscientific methods in marketing and management (Cropanzano and Becker, 2013; Ashkanasy, Becker, and Waldman, 2014).

\subsection{Organization Science}

Organizational science is loosely defined as the set of disciplines that study the functioning of human organizations and their well-being. It is an interdisciplinary field, including human resources management, industrial and organizational psychology, organizational behavior, organizational theory, strategic management, and management. It also draws from other social science disciplines such as psychology, sociology, political science, economics, and anthropology. Thus, any study of how human organizations function can fall under the purview of organizational science.

Of particular importance to organizational neuroscience is the field of organizational behavior, which refers to the study of human behavior in organizations. Organizational science does not face the same problem as economics insofar as it does not assume that humans are rational. In fact, it has always given a prominent role to subjective aspects of human behavior and decision making such as emotions and moods. Organizational scholars and psychologists have long contended that behavior in 
organizations results from the interplay between the individual and the environment (Lewin, 1947). Therefore, the behavior displayed cannot be analyzed independently of the context in which it occurred. Issues related to decision making, social perceptions, moods and emotions, organizational change, creativity and innovation, culture, personality, attitudes, and behaviors are the focus of research in organization science.

As illustrated in Figure 1.1, the disciplines contributing to organizational neuroscience overlap but also focus on particular issues that are not always studied by organizational scholars. For example, neuroscientists are not concerned with the application of their findings to organizations. The same is true for neuroeconomists, social cognitive neuroscientists, and cognitive psychologists who are not necessarily concerned with the application of their findings to the workplace. However, their findings could be used by organizational scholars to understand organizational phenomena and provide guidelines for practitioners. A recent discipline that has taken advantage of the increased development in neuroscience is neuroeconomics (Camerer, 1999; Camerer and Loewenstein, 2004). Although still an emerging discipline, neuroeconomics could provide valuable insights for organizational neuroscience scholars to help them navigate the difficulties that they may face as they transform organizational neuroscience into a legitimate discipline in the organizational sciences.

\subsection{Neuroeconomics}

Neuroeconomics refers to the study of the impact of the brain on economic decisions (Glimcher, 2003; Camerer, Loewenstein, and Prelec, 2004; Zak, 2004; Innocenti and Sirigu, 2012). It is interdisciplinary in that it combines neuroscientific measurement techniques and economic methods and theory to investigate how the human brain generates decisions in economic and social contexts (Zak, 2004; Braeutigam, 2005; Fehr, Fischbacher, and Kosfeld, 2005). After all, the human mind is the driver of all economic action (Braeutigam, 2005) and any study of human economic behavior cannot ignore the brain.

One of the goals of neuroeconomics is to explore the unobservable, subjective aspects of decision making in economic situations (Camerer, Bhatt, and Hsu, 2007), thereby overlapping with fields such as psychology and organizational behavior. Some of the pioneers of neuroeconomics, such as Camerer et al. (2004), contend that "in a strict sense, all economic activity must involve the human brain" (p. 555). Neuroeconomics is a subfield of behavioral economics and experimental economics - it addresses the neural foundations of behaviors that 
behavioral economists often study; it also involves elaborate experiments using neuroscientific tools to study economic phenomena. For instance, issues of revealed preferences, time discounting, and valuation, to name but a few, are studied using neuroscience methods.

Neuroeconomics also uses game theory (Von Neuman and Morgenstern, 1944; Camerer, 2003) to study phenomena such as trust, cooperation, fairness, and others. The most popular games used in neuroeconomics research are the Ultimatum Game (Güth, Schmittberger, and Schwarze, 1982; Thaler, 1988), the Prisoner's Dilemma Game (Fehr and Camerer, 2007; Sanfey, 2007), and the Trust Game (Kreps, 1990; Berg, Dickhaut, and McCabe, 1995; Johnson and Mislin, 2011).

The Ultimatum Game includes two players: a proposer and a responder. The first player, the proposer, receives a sum of money, generally $\$ 10$ dollars in most experiments, and decides how to split it between him- or herself and the other player, generally known as the responder. The responder may decide to accept or reject the offer. If the offer is accepted, the money is split according to the proposal. However, if the offer is rejected, both players end up empty-handed. This game is used in experimental economics, behavioral economics, and now neuroeconomics to study issues of fairness. Very often, responders reject offers less than 30 percent of the amount.

In the Prisoner's Dilemma Game, two prisoners are arrested for a crime allegedly committed. The two prisoners are in separate cells and do not have any means to communicate between them. Because the prosecutor lacks sufficient evidence for a conviction of the two prisoners, he or she approaches them individually and offers a bargain. The prosecutor gives each prisoner the opportunity either to betray the other by testifying that the other committed the crime, or to cooperate with the other by remaining silent. Each prisoner is faced with three choices: (1) if prisoner $\mathrm{A}$ and prisoner $\mathrm{B}$, each betray the other, each of them serves two years in prison; (2) if prisoner A betrays prisoner B but prisoner B remains silent, prisoner A will be set free and prisoner $B$ will serve three years in prison (and vice versa); (3) if prisoner A and prisoner B both remain silent, both of them will only serve one year in prison (on the lesser charge). Very often, each prisoner decides to betray the other, when in fact each would be better off by cooperating. The matrix displayed in Table 1.1 illustrates the possible outcomes of the Prisoner's Dilemma Game. There are several versions of the game, which is often used in behavioral economics and neuroeconomics to study cooperation and reciprocity. 


\section{Table 1.1 Payoffs in the Prisoner's Dilemma Game}

\begin{tabular}{lll}
\hline & Prisoner B Cooperates & Prisoner B Betrays \\
\hline Prisoner A Cooperates & $\begin{array}{l}\text { Each prisoner serves one } \\
\text { year }\end{array}$ & $\begin{array}{l}\text { Prisoner A gets three } \\
\text { years' sentence } \\
\text { Prisoner B goes free }\end{array}$ \\
\hline Prisoner A Betrays & $\begin{array}{l}\text { Prisoner A goes free } \\
\text { Prisoner B gets three } \\
\text { years' sentence }\end{array}$ & $\begin{array}{l}\text { Each prisoner gets two } \\
\text { years' sentence }\end{array}$ \\
\hline
\end{tabular}

Berg et al. (1995) developed the Trust Game, which is played as follows. Subjects in Room A decide how much of their $\$ 10$ show-up fee to send to an anonymous counterpart in Room B. Subjects are informed that each dollar sent would triple by the time it reaches Room B. Subjects in Room $\mathrm{B}$ then decide how much of their tripled money to keep and how much to send back to their respective counterparts. The unique Nash equilibrium prediction for this game with perfect information is to send zero money. However, the authors found that this prediction was rejected because 30 out of 32 Room A subjects sent money. This contradiction could be explained by the fact that trust is an important component of exchange relationships. The trust game is used to study trust, cooperation, reputation, and reciprocity.

Sanfey (2007) argues that by combining the models and tasks of game theory with modern psychological and neuroscientific methods, the neuroeconomic approach to the study of social decision making has the potential to extend our knowledge of brain mechanisms involved in social decisions and to advance theoretical models of how we make decisions in a rich, interactive environment. Lee (2008) emphasized the use of game theory in neuroeconomics by arguing that decision making in a social group has two distinguishing features. First, humans and other animals routinely alter their behavior in response to changes in their physical and social environment. As a result, the outcomes of decisions that depend on the behavior of multiple decision makers are difficult to predict and require highly adaptive decision making strategies. Second, decision makers may have preferences regarding consequences for other individuals and therefore choose their actions to improve or reduce the well-being of others.

According to Camerer (2007), the largest innovation may come from pointing to biological variables that have great influence on behavior and are underweighted or ignored in standard economic theory. The neuroeconomic theory of the individual replaces the (perennially useful) fiction 
of a utility-maximizing individual who has a single goal with a more detailed account of how components of the individual - brain regions, cognitive control, and neural circuits - interact and communicate to determine individual behavior (p. C28).

Neuroeconomics has primarily challenged the standard economic assumption that decision making is a unitary process, a simple matter of integrated and coherent utility maximization, suggesting instead that it is driven by the interaction between automatic and controlled processes (Loewenstein, Rick, and Cohen, 2008). Volk and Köhler (2012) argue that neuroeconomics can help researchers "form better theories about the underlying reasons for observable behaviors in a given context and create more accurate tests of their theories than if they were to use other, less direct measures such as standard self-report measures" (p. 523). By doing so, neuroeconomics can contribute to theory pruning (Leavitt, Mitchell, and Petterson, 2010).

The same reasoning could apply to organizational neuroscience. Indeed, neuroscience could help prune theories in organizational sciences. Neuroeconomics studies phenomena that are relevant for organizational neuroscience. For example, neuroeconomists study topics such as decision making, uncertainty, risk, trust, and cooperation, even the emotional basis of human behavior. Findings from neuroeconomists could find applications in organizations. Indeed, organizations are entities populated by people who make decisions on a regular basis. In addition, organizational members cooperate with one another to accomplish certain tasks. Hence, there should be a close collaboration between neuroeconomists and organizational neuroscience scholars.

\subsection{Social Cognitive Neuroscience}

Social cognitive neuroscience is closely related to social neuroscience, and is defined as "an interdisciplinary field devoted to understanding how biological systems implement social processes and behavior, capitalizing on biological concepts and methods to inform and refine theories of social processes and behavior, and using social and behavioral concepts and data to refine theories of neural organization and function" (Cacioppo et al., 2007, p. 100). According to Lieberman (2007a), social cognitive neuroscience examines social phenomena and processes using cognitive neuroscience research tools such as neuroimaging. Knowledge from social cognitive neuroscience helps to accomplish the following: (1) understanding others; (2) understanding oneself; (3) controlling oneself; and (4) the processes that occur at the interface of self and others. 
However, the field of social neuroscience could not ignore the role of social cognitions in social and interpersonal relations. Thus, the field of social cognitive neuroscience developed in large part as a recognition of the role of social cognitions in social neuroscience. Social cognition refers to "the ability to construct representations of the relations between oneself and others, and to use those representations flexibly to guide social behavior" (Adolphs, 2001, p. 231). Hence, it encompasses any cognitive process that involves conspecifics, either at a group level or on a one-to-one basis (Blakemore, 2004, p. 216). This is particularly relevant to organizations because they represent social entities where people interact with one another on a regular basis.

Social cognitive neuroscience is a multidisciplinary field embedded in the social sciences because it draws from social cognition, political cognition, behavioral economics, and anthropology (Lieberman, 2007a). It allows people to understand themselves and others (Lieberman, 2007a). Lieberman focuses on social cognitive neuroscience as a means of studying social behavior using the tools of cognitive neuroscience, a field that combines cognitive psychology and neuroscience. Social cognitive neuroscience combines the "tools of cognitive neuroscience with questions and theories from various social sciences" (Lieberman, 2007b, p. 260).

According to Bechtel (2002, p. S49), the dominant perspective in cognitive neuroscience revolves around two principles: (1) different brain areas perform different information processing operations and (2) an explanation of a cognitive performance involves both decomposing an overall task into component information processing activities and determining what brain area performs each. Ochsner and Lieberman (2001) consider social cognitive neuroscience as "an emerging interdisciplinary field of research that seeks to understand phenomena in terms of interactions between 3 levels of analysis: the social level, which is concerned with the motivational and social factors that influence behavior and experience; the cognitive level, which is concerned with the information-processing mechanisms that give rise to social-level phenomena; and the neural level, which is concerned with the brain mechanisms that instantiate cognitive-level processes" (p. 717, abstract).

Ochsner and Lieberman (2001) represented these three levels of analysis by a prism (Figure 1.2) intended to capture the idea that social cognitive neuroscience is about studying phenomena at many levels of analysis to learn how and when brain systems are used to mediate motivated human behavior (Ochsner and Lieberman, 2001, p. 719). The task of cognitive neuroscience is to map the information-processing structure of the human mind, and to discover how this computational organization is implemented in the physical organization of the brain. It 


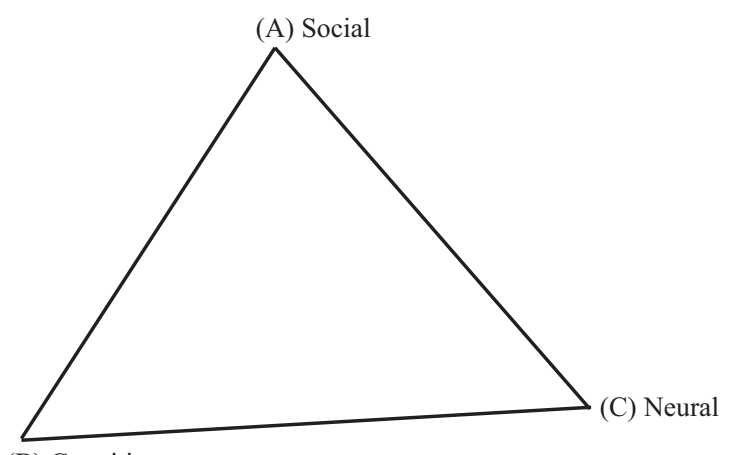

(B) Cognitive

\section{Figure 1.2 The prism of social cognitive neuroscience}

helps address questions such as, how do brain events give rise to mental and behavioral events? Because organizations are populated by people and involve their interactions, findings from social cognitive neuroscience could enrich organizational neuroscience by providing knowledge related to the neural basis of social and interpersonal relations.

Because social cognitive neuroscience uses neuroscience and cognitive psychology to study social behavior, it has implications for social behavior in organizational settings. Subsequently, Butler and Senior (2007) and Lee and Chamberlain (2007) have expanded it to organizational contexts. For proponents of a social cognitive neuroscience perspective of organizational behavior, human behaviors in organizations can better be understood if one makes sense of their neural foundations. Such an approach offers some interesting insights because knowledge of the brain can provide useful information about how people react toward others and understand the organizational world they navigate on a regular basis. For example, organizations can be perceived as arenas of emotionproduction; that is, people act and react to others and events based on their emotional appraisal.

We know that emotions play a critical role in how people make decisions and act in organizations. This is hardly new, but social organizational neuroscience can provide further refinements. Thus, organizational neuroscience draws from social cognitive neuroscience insofar as it acknowledges the role of the neural basis of cognitions in organizational behavior. It can build on previous research on social cognitive neuroscience and apply it to organizational life. Organizational neuroscience builds on the scientific developments in organizational behavior and the recognition of the role of cognition and emotion in explicating human behavior at work. 


\subsection{Cognitive Psychology}

Cognitive psychology is the study of how people mentally represent and process information. As such, it includes within its domain mental abilities such as perception, learning, memory, reasoning, problem solving, and decision making (Sternberg, 1981, p. 1181). The focus on cognitions had led to the development of theories on the role of cognition on decision making under risk and uncertainty. It has also led to the development of new theories such as prospect theory (Kahneman and Tversky, 1979) and cognitive biases in decision making (Tversky and Kahneman, 1974).

Cognitive psychology could play an important role in organizational neuroscience for at least three reasons. First, the cognitive revolution in psychology has allowed a focus on cognitions and thoughts (Sperry, 1993; Miller, 2003). In fact, how people react to situations depends on how they construe them. In this approach, mental abilities are considered key factors in influencing human behavior. Cognitive psychology contributed to the development of artificial intelligence (Newell and Simon, 1976; Simon, 1980). Computers could replicate human thoughts and make decisions. One of the limitations of previous theories of cognitive psychology was the role of emotions in human actions. Because cognitions could not be integrated smoothly with emotions, scholars introduced the construct of embodied cognition, which focuses on the importance of action and on how action shapes perception, the self, and language (Glenberg, Witt, and Metcalfe, 2013).

Second, the development of the construct of socially situated cognition (Neisser, 1967; Sperry, 1993; Mandler, 2002; Miller, 2003; Smith and Semin, 2004) indicates that organizational phenomena could be studied through the lens of cognition, behavior, and context. Smith and Semin (2004, p. 53) highlight four assumptions that are common to socially situated cognition: (1) cognition is for the adaptive regulation of action, and mental representations are action oriented; (2) cognition is embodied, drawing on our sensorimotor abilities and environments as well as our brains; (3) cognition and action are the emergent outcome of dynamic processes of interaction between an agent and an environment; and (4) cognition is distributed across brains and the environment.

Third, most of the topics studied in organizational science involve the processing of information: "All human mental events occur as the result of neural information processing" (Ilardi and Feldman, 2001, p. 1072). This statement may imply that the organizational neuroscience paradigm suggests that human behaviors in organizations occur as the result of neural information processing. For example, people constantly make 
decisions that are choices based on how they evaluate the situation and the resources available. People also decide who to trust or not. These examples demonstrate the potential contribution that cognitive psychology could make to organizational neuroscience.

Cognitive psychology helps us to understand the mental structure of thoughts and human behavior. Poldrack (2006) argued that "the goal of cognitive psychology is to understand the underlying mental architecture that supports cognitive functions" (p. 59). As a result, most research in cognitive psychology involves the manipulation of tasks to determine which cognitive functions are affected. Cognitive psychologists also explore the types of cognitive functions and abilities that can help individuals effectively perform certain tasks. As Poldrack notes, "if neuroimaging were able to provide information regarding what cognitive processes were engaged in performance of a particular task, cognitive psychologists would have gained a powerful new tool" (ibid.). Cognitive psychology is also concerned with the role of automatic and controlled processing in cognition and its impact on human behavior (Sternberg, 1981; Lieberman, 2007a, 2007b).

\section{LEVELS OF ANALYSIS OF ORGANIZATIONAL NEUROSCIENCE}

\subsection{Individual Level of Analysis}

If organizational neuroscience is to become a legitimate field of study in the organizational sciences, we must identify the levels of analysis it could address. Should it focus only on the individual level of analysis or should it also address group and/or organizational levels of analysis? (See Figure 1.3, which illustrates all three.) Beugré (2010) suggests that neuro-organizational behavior includes three levels of analysis: neural, cognitive, and behavioral. The neural level focuses on identifying the brain regions that are activated when organizational members display particular types of behavior. The cognitive level involves internal mental processes that rely on these neural substrates such as memory and information processing (Lee and Chamberlain, 2007). The behavioral level concerns observable actions displayed by organizational members.

The neural level deals with how different brain structures function and interact to influence human attitudes and behaviors. Questions related to the role of specific brain structures could be relevant here. This is important because one needs to clearly understand the specific role of particular brain structures before identifying which ones influence the 
behavior of interest. At the cognitive level, researchers are interested in knowing how brain structures affect the thought processes we experience. Such an understanding could provide clues for knowing how people process information and make decisions. The behavioral level is the observable part and can help us understand how we act or react in particular ways when faced with some environmental stimuli. All three levels focus on the behavior of individuals acting independently. However, we know that in organizations, people tend to work in groups and teams to perform specific tasks. Can organizational neuroscience address the functioning of such units of analysis?

\subsection{Group and Social Interaction Level of Analysis}

At this level, organizational scholars might look at the impact of neuroscience on group functioning and social interactions within organizations. Understanding of neural science concepts and methods could help explain how interpersonal dynamics occur in organizations. For example, issues of hidden biases and diversity could be studied to understand whether they are rooted in brain structures. Likewise, topics such as social cognition, shared cognitions, shared mental models, and intergroup relationships, including intergroup conflicts, could be studied through the lens of neuroscience. The discovery of the mirror neuron system, a group of specialized neurons that mirrors the actions and behavior of others (Rizzolatti and Craighero, 2004; Rizzolatti and FabbriDestro, 2008), could help shed light on the neural basis of social interactions in organizations.

\subsection{Organizational Level of Analysis}

Research in organizational behavior focusing on the level of the entire organization has included topics such as organizational politics, organizational structure and design, organizational culture, and change. An organizational-level analysis of organizational neuroscience could entail the study of the neural basis of the topics identified above. For example, researchers could study the neural basis of organizational culture, change, or political behavior in organizations. In fact, understanding the neural underpinnings of behavior can help explain group- or organizational-level phenomena because "many phenomena in organizations have their theoretical foundation in the cognition, affect, behavior, and characteristics of individuals, which, through social interaction, exchange, and amplification, have emergent properties that manifest at higher levels. In 
other words, many collective constructs represent the aggregate influence of individuals" (Kozlowski and Klein, 2000, p. 15).

\begin{tabular}{|c|c|c|}
\hline & & Organizational Level \\
\hline & Group Level & $\begin{array}{l}\text { Neural basis of culture } \\
\text { Neural basis of change } \\
\text { Neurostrategy } \\
\text { Neural basis of organizational } \\
\text { politics }\end{array}$ \\
\hline Individual Level & $\begin{array}{l}\text { Neural basis of shared mental } \\
\text { models } \\
\begin{array}{l}\text { Neural basis of emotional } \\
\text { contagion }\end{array}\end{array}$ & \\
\hline Individual neurons & Mirror neurons & \\
\hline Neural networks & $\begin{array}{l}\text { Neural basis of interpersonal } \\
\text { trust }\end{array}$ & \\
\hline $\begin{array}{l}\text { Neural basis of individual } \\
\text { motivation }\end{array}$ & & \\
\hline $\begin{array}{l}\text { Neural basis of individual } \\
\text { decision making }\end{array}$ & & \\
\hline Neural basis of emotions & & \\
\hline Neural basis of personality & & \\
\hline $\begin{array}{l}\text { Neural basis of ethics and } \\
\text { morality }\end{array}$ & & \\
\hline
\end{tabular}

Figure 1.3 Levels of analysis of organizational neuroscience

\section{PROSPECTS FOR ORGANIZATIONAL NEUROSCIENCE}

What questions can organizational neuroscience address? What topics can better be studied as a result of using neuroscientific tools and techniques? How can an understanding of neuroscience lead to better management practices? This section addresses these questions.

\subsection{Contribution to Research}

Organizational neuroscience can contribute to theory development and the analysis of organizational topics in a new light. For example, topics currently studied in the organizational sciences such as leadership, motivation, decision making, trust, creativity, and change could be 
expanded with neuroscientific tools. The use of these tools could help better understand these organizational phenomena.

Becker and Cropanzano (2010) suggested three areas where organizational neuroscience could provide useful insights to organizational scholars: (1) combatting procrastination; (2) mirror neurons and group subclimates; and (3) attitudes and behaviors. To explain the role of organizational neuroscience in combatting procrastination, the authors use the dual-process theory (implicit, automatic processing and explicit, controlled processing). Humans are vulnerable to procrastination because of the existence of an automatic system that tends to retain them in the status quo and away from new targets and behaviors. The prefrontal cortex plays an important role in the control system that allows for the planning and deliberate focus on activities.

The mirror neuron system (MNS) presents evidence that people unconsciously react to the actions of others and could play a central role in social interactions and teamwork in organizations. According to Rizzolatti and Craighero (2004) and Rizzolatti and Fabbri-Destro (2008), the mirror neuron system responds to visual observation and facial expressions. People tend to mimic actions performed by others. Hence, this system could help to advance social learning and explain vicarious learning. People learn by observing others. Becker and Cropanzano (2010, p. 1057) contend that "when team members interact, the MNS is finely tuned to perceive the actions, expressions, and body language of others. They implicitly learn from and assess the behaviors of other members. The MNS will lead group members who interact frequently to converge toward attitudes and behaviors that are adaptive for the group, but not necessarily for the organization." In other words, the mirror neuron system could explain how good or bad behavior spreads within teams and even organizations.

Becker and Cropanzano (2010) also mentioned the role of neuroscience in explaining how implicit attitudes can influence explicit attitudes and behaviors in organizations. Using the dual-process theory, they contend that "an implicit attitude is rapid, automatic, and comprises unconscious evaluative response to stimuli, whereas an explicit attitude is a relatively slower, deliberative conscious evaluation based on contextual information" (p. 1058).

Other authors have also emphasized the potential benefits of organizational neuroscience (Beugré, 2010; Powell, 2011; Ward and Becker, 2013). Ward and Becker (2013) identified four benefits of organizational neuroscience: (1) it offers several tools for data collection; (2) it offers alternatives to self-report questionnaires; (3) it offers opportunities for 
strengthening construct validation; and (4) provides new ways to refine existing theories.

\subsection{Contribution to Management Practice}

An understanding of organizational neuroscience could provide guidelines for management practice in several areas. For example, Ward, Volk, and Becker (2015) identified several applications of organizational neuroscience, including training and development, job design, highperformance assessment, motivating communication, and conflict prevention. It is worth mentioning that several domains could be added to those identified by Ward and collaborators. For instance, one could use findings from organizational neuroscience to further creativity, team building, emotional control and regulation, the development of trust, decision making, ethical behavior, and diversity in the workplace. In fact, the remaining chapters of this book, after discussing the research tools in the next chapter, deal with the role of neuroscience in understanding these topics and providing guidelines for management practice.

Rangel, Camerer, and Montague (2008) note that organizational neuroscience can be useful to artificial intelligence: "A question of particular interest is which features of the brain's decision-making mechanisms are optimal and should be imitated by artificial systems, and which mechanisms can be improved on" (p. 555). In an era of big data analytics and algorithms, findings from organizational neuroscience could provide useful guidelines for managers and organizations. It could also be useful for self-development. Indeed, individuals could use findings from brain science to improve their lives as individuals and economic agents. There is evidence that exposure to brain functioning and especially one's own brain images tends to improve self-regulation: "Neuroscience technology, combined with brain education and training, can improve self-control at its source in the brain, and improve experiences, expressions and effectiveness" (Powell and Puccinelli, 2012, p. 210).

Despite its potential benefits, organizational neuroscience still faces several challenges. These challenges are often related to the tools used by researchers. The following chapter discusses neuroscientific tools that are relevant for research in organizational neuroscience. It will also address the challenges organizational neuroscience faces as a nascent discipline. 